\title{
Digital humanities and practical memory: modelling textuality
}

\author{
Arianna Ciula \\ King's Digital Lab, King's College London \\ E-mail: arianna.ciula@kcl.ac.uk
}

\begin{abstract}
This paper reflects on the relation between Humanities and Digital Humanities from scholarly perspectives intertwined as they are with political stances. Often characterized as enabling a unifying as well as transformative sentiment for the Humanities as a whole, Digital Humanities can be described as a challenging hybridization of scholarly practices contingent to social and cultural contexts. The core of the paper relates to one of the topics of the 2015 conference on Rethinking Humanities, namely digital memory, by recalling on the concept of Humanities as scholarship engaged with meaning-making prac-

literature and some co-authored research ${ }^{1}$, I present a humanities-informed theorization of modelling in Digital Humanities as a meaning-making practice enacted in the present and aiming at repurposing the past. As privileged objects of digital modelling activities, texts are repurposed via the creation and manipulation of (digital) external representations. An informed theory of textuality reminds us how cultural products embed the processes of their creations and uses. Can digital models enact such practical memory and become in themselves strategies to exercise memory and encode knowledge?
\end{abstract} tices connecting past and present. Building on the

\section{Digital humanities representing and transforming the humanities?}

THE perspective of the conference this paper contributed to departed from an idea of Humanities as a whole, a unifying concept that Digital Humanities in itself contribute to bring to the fore. This idea of Humanities as a unifying concept could summarize in fact "what is new in the humanities today". ${ }^{2}$ On the other hand, the fragmentation of disciplines within the Humanities is

Part of the work presented in this article was conducted while the author was based at the Department of Humanities, University of Roehampton, UK (until January 2017).

A Revista Estudos em Comunicação é financiada por Fundos FEDER através do Programa Operacional Factores de Competitividade - COMPETE e por Fundos Nacionais através da FCT - Fundação para a Ciência e a Tecnologia no âmbito do projeto Comunicação, Filosofia e Humanidades (LabCom.IFP) UID/CCI/00661/2013.
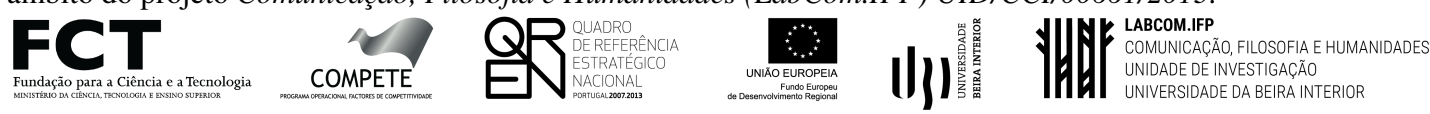

1. This research was part of The work for this essay was conducted as part of the research project Modelling between digital and humanities: thinking in practice (www.modellingdh.eu). Project partners: Arianna Ciula, University of Roehampton (until January 2017) and King's Digital Laboratory (King's College London, from February 2017); Øyvind Eide, University of Cologne; Cristina Marras, Institute for European Intellectual Lexicon and History of Ideas, National Research Council (Rome); Patrick Sahle, University of Cologne. The project is funded from April 2016 to March 2018 under the Volkswagen Stiftung programme: "Original - isn't it?"New Options for the Humanities and Cultural Studies, Funding Line 2 "Constellations"(2016-2017).

2. This is a quote from the rationale of the conference (International Conference Rethinking Humanities 2015). 
a relatively recent phenomenon dating back to the nineteenth century, when the reform of Universities and the formation of modern scientific disciplines took place. This process sealed "the fate of the "humanist mathematics" and marked "a break with the classics" (Bod, Maat and Weststeijn 2012, 12).

Since the nineteenth century the humanities have typically been defined as those disciplines that investigate the expressions of the human mind (Geisteswissenschaften). Thus, the study of music, literature, language, visual arts all belong to the realm of the humanities, in contrast to the study of nature which belongs to the domain of the natural sciences. And the study of humans in their social context belongs to the social sciences. But these definitions are unsatisfactory. Mathematics is to a large extent a product of the human mind, and yet it is not considered a humanities discipline. A pragmatic stance may be more workable: the humanities are those disciplines that are taught and studied at the various humanities faculties. [...] While various histories of some single humanistic disciplines have been written, such as the history of linguistics or the history of literary theory, connections between methods and principles in literary theory and those in art history or between musicology and linguistics are rarely made - perhaps because of the notorious fragmentation of the humanities during the last century. (Bod, Maat and Weststeijn 2010, 7-8)

Notwithstanding its pragmatic stance, a unifying concept of the Humanities recalls the earlymodern European context, when humanistic scholarship intertwined naturally with formal and empirical studies. ${ }^{3}$ In addition, it could be argued that the use of the term Humanities also reinforces a sense of global (e.g. Bod et al. 2012, 13) and of public humanities. Referring to the Humanities as one pragmatic concept could serve the wishful and fruitful aim of overcoming fragmentation, of reconnecting two or more epistemic cultures as well as the aim of bridging geopolitical borders of scholarship and the enclosed academic towers with the public spaces. Nevertheless, a Humanities projected to the future as well as to the past can easily be steered ideologically towards a simplistic or even positivist and homogenizing agenda (Svensson 2012). ${ }^{4}$

Hence, while keeping an historically-aware pragmatic perspective, it is useful to recall a broad definition of Humanities based on its content of study:

3. Cfr. Bod, Maat and Weststeijn (2010, 12-13): "Together, the seven parts of this book illustrate the width and depth of the history of the humanities in early modern Europe, as well as their mutual intertwining and connection with the exact sciences. The humanities instigated a new secular world view (Steenbakkers, Leezenberg, Hanegraaff, Gatti), they rebutted forgeries that no-one dared to question before (Pyle, Steenbakkers, P.charman), and with their standard of precision, consistency and criticism (Pyle, J.nsson, Rowland, Cohen, Groenland), the humanities deeply influenced the exact sciences (Pyle, Cram, Maat, Cohen). [...] Their relation with the New Sciences indicates that the humanities not only preceded the sciences but also shaped them to a very large extent via the formal and empirical study of music, art, language and texts (Cohen, Rowland, Pyle, Weststeijn, Cram, Maat)."

4. Svensson warns against this: "While the ideas of grand challenges and big humanities certainly have attraction and require essential forward thinking in order to identify complex problems and large-scale visions, we should be careful not to uncritically accept the frame of big humanities, which, for instance, has a tendency to be coupled with a positivist agenda and a homogenization of the humanities (cf. [Scout 2006]).” (Svensson 2012) 
The humanities study the meaning-making practices of human culture, past and present, focusing on interpretation and critical evaluation, primarily in terms of the individual response and with an ineliminable element of subjectivity. (Small 2013, 57) ${ }^{5}$

I will not dwell on this definition at this point, but I would like to highlight two concepts which are at the core to my argument, namely, 'meaning-making practices' and 'subjectivity'.

The Digital Humanities have been contributing to the unifying yet pragmatic concept of the Humanities not only in virtue of its denomination, but also by "representing or manifesting the humanities": 6

[...] the digital humanities often becomes a laboratory and means for thinking about the state and future of the humanities [...] regardless of the variety of digital humanities, there is often an actual or presumed engagement with all or most of the humanities. This gives the digital humanities more reach than most regular departments, disciplines and centers, and arguably, both an interest and a mandate to be invested in the future of the humanities at large (somewhat like humanities centers). The fact that the field tends to be institutionalized differently than other academic enterprises might also help in the sense that it facilitates a freer role and possibly a less competitive stance in relation to established departments and disciplines. [...] Indeed, research infrastructure has cross-sectional potential, and there is often at least nominal interest in including in the humanities in new research infrastructure initiatives ([Svensson 2011]). Here the digital humanities matches the expectations more than in most other areas. This may lead to the digital humanities representing the humanities in relation to other areas of research and development such as science and engineering, which in turn helps create interest for the field outside of the humanities and contributes to the sense of digital humanities as representing or manifesting the humanities. (Svensson 2012)

No doubt, when this representative role is perceived as a self-election from the part of those engaged in Digital Humanities, tensions with the Humanities that do not recognize themselves in this representation emerge and have regularly found expressions in various forms and formats. Besides its more or less contested advocacy role in the name of the Humanities, what is of interest to us here is that the Digital Humanities has being characterized mainly as aiming at transforming the Humanities, in particular, because of their need of extensive technical infrastructures (Svensson

5. Small (2013) unpacks further this definition as following: "In the main the humanities value qualitative above quantitative reasoning; they place greater faith in interpretative than in positivistic thinking; unlike the sciences and the scientific wing of the social sciences they do not have a dominant methodology, and many of their truth claims are not verifiable as those of the natural sciences are verifiable; they tend, accordingly, to distrust proceduralism and to value independence of thought. They are orientated as much toward historical analysis as toward synchronic structural analysis, and as much toward the medium of expression as towards its content (tending to see the form/content distinction as itself problematic). They attend to the role of the perceiver in ascertaining even the most philosophically secure of knowledge claims; and they have an interest, often they also take pleasure, in the specificity of the object of study and the specificity of the individual response (its content and its style) over and above the generalized or collective response. Not least, they respect the products of past human endeavours in culture, even when superseded." (Small 2013, 57)

6. See for example (4Humanities). 
2012), ${ }^{7}$ but also, at a deeper level, because of their acting as a "disruptive political force that has the potential to reshape fundamental aspects of academic practice" (Gold 2012), ${ }^{8}$ including the mechanisms for the evaluation of scholarship (Nowviskie 2012). The potential transformative function of Digital Humanities is explained as the ability to question and even hack the "scholarly infrastructure" as a whole, and to channel a common "transformative sentiment" (Svensson 2012). One could argue that such transformative sentiment is destined to diffuse in the ongoing move of the Digital Humanities from "institutionally peripheral position" in academia (Svensson 2012) to institutionalized subject, hence the question mark in the title of this section.

From the Digital Humanities perspective, what is new (and old) in the Humanities today is a not unproblematic yet interesting blending and hybridization ${ }^{9}$ of scholarly changes with social and cultural ones ${ }^{10}$. To sum up, this blending and hybridization entail at least the following: the blurring of research and publishing processes (Svensson 2012); the emergence of "trading zones" between research, education and other activities often in the name of public humanities (Svensson 2012); the bridging of relatively recent divide across scientific cultures and epistemic traditions; the practicing of collaboration and "mutual respect" (Svensson 2012) across a diversity of epistemic traditions and professional status.

\section{Between digital and humanities}

Against this background of characterizations that undoubtedly benefit from evidence but bear also rhetorical power, to set Digital Humanities within its context of emergence and establishment, I would like to focus mainly on its research agenda being of the Humanities. In particular, with respect to the topic of digital memory, I would like to draw attention to the "modes of engage-

7. "[...] some digital humanities work requires extensive technology infrastructures, which is not very common in the humanities. Based on these and other factors, there is a strong sense that the university and the humanities need to change to accommodate this type of work, and all this feeds into a vision of a transformed humanities." (Svensson 2012)

8. Here is the full quote of this same passage: "At stake in the rise of the digital humanities is not only the viability of new research methods (such as algorithmic approaches to large humanities data sets) or new pedagogical activities (such as the incorporation of geospatial data into classroom projects) but also key elements of the larger academic ecosystem that supports such work. Whether one looks at the status of peer review, the evolving nature of authorship and collaboration, the fundamental interpretive methodologies of humanities disciplines, or the controversies over tenure and casualized academic labor that have increasingly rent the fabric of university life, it is easy to see that the academy is shifting in significant ways. And the digital humanities, more than most fields, seems positioned to address many of those changes. [...] Indeed, fault lines have emerged within the DH [Digital Humanities] community between those who use new digital tools to aid relatively traditional scholarly projects and those who believe that DH is most powerful as a disruptive political force that has the potential to reshape fundamental aspects of academic practice" (Gold 2012).

9. I use the word hybridization as instance of a new discipline borne as a hybrid in the same way as Kartsten used this term while reflecting on the formation of comparative linguistics: "The new hybrid reflects ideas from the culture in which it emerges, the values that reign supreme at the place of its emergence (including its specific institutional setting) and from the various fields of study it has borrowed ideas from. All these aspects are put in a mixer and the new substance coming out of it is the new discipline." (Karstens 2012, 105)

10. For an extensive argument on the connections between digital modernity and the present and future of digital humanities see Smithies 2017. 
ment between the digital and the humanities" (Svensson 2012) ${ }^{11}$ which call for a "humanistically informed theory" (Drucker 2012). ${ }^{12}$ In doing this, my aim is to contribute to the understanding of how essential "apprenticeship" in the Digital Humanities - what we might call more broadly learning by doing or understating technology by using and developing it - can lead to a "humane project" (McCarty 2012,39) and hence fight an apocalyptic picture whereby those of us who are engaged with Digital Humanities research lose sight of what we want the technology for. ${ }^{13}$ The possibly most productive concept aiming at describing and theorizing the practices of doing research and teaching in Digital Humanities is modelling. By modelling I intend mainly the creation and manipulation of external representations, encompassing the whole spectrum of what Bradley (2015) calls tools for making, for exploring and for thinking. Building on previous co-authored research (Ciula and Eide 2014, Ciula and Marras 2016, Ciula and Eide 2017), what I claim in this paper is that modelling can be considered both a meaning-making practice and a strategy to exercise individual and collective (active) memory. To substantiate my claim I chose to focus on textuality - hence on the sociology of the textual condition and the engagement with the digital as an ongoing "repurposing of the work of the past" (McGann 2014, 46) - as well as on the theory of modelling. With respect to cultural memory the ESF Science Policy Briefing Cultural Literacy in Europe today states that:

What is perceived as history varies according to the way memory is shaped through different techniques of remembering: whether it is passed on orally or written down, what elements are deemed important and what are left out and how facts are interpreted in their relationship to each other. [...] both individually and collectively, remembering and forgetting are only made possible by the use of 'cultural tools'. [...] What impact does the advent of new technologies have on such regimes of memory [...]?" (ESF-COST 2013, 7-8)

Our tools for memory are many and various. In the context of Digital Humanities one fruitful way to explore the mutual impact between technology and regimes of memory is to reflect on how digital scholarship is producing new intermediaries to the written past. For example, with

11. Cfr. Svensson (2012): "Looking at the landscape of the digital humanities more broadly, it seems tenable to assume that the most far-reaching employment of the digital as a means of (re)negotiating the humanities does not come from [...] primary instrumental orientation, nor from internet studies and many other cultural studies approaches to the digital with their primary interest in the digital as an object of analysis (and a stronger disciplinary anchoring). Rather, it seems that approaches and initiatives invested in several modes of engagement between the digital and the humanities are more likely to relate to the place and future of the humanities."

12. "I suggest that it is essential if we are to assert the cultural authority of the humanities in a world whose fundamental medium is digital that we demonstrate that the methods and theory of the humanities have a critical purchase on the design of platforms that embody humanistic values. [...] The challenge is to shift humanistic study from attention to the effects of technology (from readings of social media, games, narrative, personae, digital texts, images, environments), to a humanistically informed theory of the making of technology (a humanistic computing at the level of design, modeling of information architecture, data types, interface, and protocols). [...] texts (in the broad sense of linguistic, visual, acoustic, filmic works) are not static objects but encoded provocations for reading. [...]." (Drucker 2012)

13. Cfr. McCarty $(2012,38)$ : “[...] many of the scholars [...], not paid to think and act like scholars, have lost sight of that which infrastructure is for." 
respect to text-based archival research conducted on documentary primary sources, certain key publications produced as part of Digital Humanities collaborative research projects that Palmer (2005) would call "access resources" have the potential to become the new intermediaries to the written record (Ciula and Lopez 2009). These publications represent an intersection between the 'new' genres of digital scholarship and the established ones. For many historians, reading and writing are the outcomes of deep inquiry over months or even years around a set of core materials. Hybrid publications in print and web forms are particularly interesting to analyze for the way their development and use enact the convergence and divergence between editorial strategies and multiple material realizations spanning different media. They are also well placed to make emerge the problematic yet revealing relation between digital and non-digital regimes of memory, what McGann calls the "skew between our traditional cultural inheritance and the emerging digital corpus." (McGann 2014, 43). By creating and using these cultural tools, we shape scholarly (hence individual but also collective) memory - certainly not a negligible professional responsibility for digital humanists to carry.

McGann believes that "Now more than ever [...] we need to understand how bibliographical technology works. Designing optimal digital environments requires it." (McGann 2014, 1). He also argues that:

[...] textual and editorial scholarship, often marginalized in humane studies as a narrowly technical domain, should be shifted back to the center of humanist attention. Understanding the technologies of book culture is the beginning of wisdom for any practical approach to the so-called digital humanities. But you can't do that well unless you have an intimate acquaintance with the scholarship of textualities. (McGann 2014, 2)

By reflecting on the hybrid publications we produce, we can attempt to elicit the rich bibliographic code of a specific print format ${ }^{14}$ and to compare it with the digital one, not simply for analytical purposes but to get intimately acquainted with the scholars' use and understanding of these materials in its combination (digital and print). The paradigm to follow can be explained again in McGann's words:

Digitizing the archive is not about replacing it. It's about making it usable for the present and the future. To do that we have to understand, as best we can, how it functioned - how it made meanings - in the past. A major task lying before us - Its technical difficulties are great - is to design a knowledge and information network that integrates, as seamlessly as possible, our paper-based inheritance with the emerging archive of born-digital materials. (McGann 2014, 22)

This process of translation (translation is indeed what he calls it) requires a "clarity of thought about textuality" (McGann 2014, 90).

14. In the case of the Henry III Fine Rolls project discussed in (Ciula and Lopez 2009), the model under examination was the modern Calendar print edition. 


\section{Modelling textuality}

Digital Humanities can reclaim a humanities-based theorization of modelling, in particular by stressing its relation to texts and theory of texts as well as to the nature of modelling as meaningmaking practice.

Models and modelling - pervasive in scientific empirical practices - are at the core of the methodological approaches of Digital Humanities research and teaching. This sharing of epistemologies enacted via processes of modelling across disciplinary borders makes Digital Humanities to a certain extent comparable to the techno-sciences and anchors Humanities scholarship to a practical dimension renewing its fifteenth century roots of empirical studia humanitatis (Bod 2014). Thus the question is: What model of modelling can be considered 'adequate' to Digital Humanities (Ciula and Marras 2016)? And in what way are Digital Humanities rhetoric and practices transformative of the way we remember our past (and hence rethink a future)?

By considering modelling as a process of signification and reasoning in action (Ciula and Eide 2014 and 2017), we can free modelling from the reductionist frame of human-machine communication and from the concerns that seem exclusive to technical implementation. One way of achieving this is contextualizing modelling within a semiotic framework, so as to consider it as a strategy to make sense (signification) via practical thinking (creating and manipulating models). Complementary to this approach, is the consideration of computational technologies as co-constitutive of cultural practices. Consequently, the study of modelling and models has to be situated within the specific digital materialities in which they are enacted, as, for example, Dourish' does in his analysis of the materialities of databases (Dourish 2014).

Kralemann and Lattmann (2013) claim that models should be understood as signs in the Peircean sense. In Peirce's seminal theory of signs, the sign is a triadic relation between a representamen (the sign from which the relation begins, sometimes also called in the literature the sign-vehicle), its object, and the interpreting thought. Often represented as a tripod where the three "composing elements" (Olteanu 2015, 127) - object, representamen and interpretant - intersect, the sign for Peirce is, first and foremost, relational. The experience of interpreting signs or signification (semiosis) is therefore intrinsically dynamic. As a consequence, a semiotic approach which considers models as signs gives high prominence to a dynamic view on models reinstating in renewed terms the value of modeling as an open process - in particular, a process of signification. ${ }^{15}$

If modelling acts as understood from a semiotic tradition are used to make sense of our cultural objects (mainly but not only texts), they are meaning-making practices and hence in themselves object of study for the Humanities as defined above. Indeed, interestingly, the modelling process in Digital Humanities is often recognised as part of what is being modelled.

15. The model relation includes the following components: a set of objects Oi=1,..,n ((Kralemann and Lattmann 2013) call this 'extension' of the model); a theory or language (what they call the 'intention' of the model) and an object Omod (its attributes define what Kralemann and Lattmann call the 'syntax' of the model). For the subject who chooses Oi and a theory or language, Omod becomes a model of the objects Oi on the basis of a representational relation between its syntax and the semantic attributes of Oi. This relation is determined by the context of a theory as well as by the purpose of the specific act of modelling. For more details on the application of this framework to modelling in Digital Humanities see (Ciula and Marras 2016; Ciula and Eide 2017). 
Texts - dynamic cultural objects contingent to the contexts of production and reading, expressed in a wide range of manifestations from linear to discontinuous narrative, from manuscripts to printed editions, encompassing hybrid modalities - are or at least have been so far the privileged objects of modelling activities in Digital Humanities.

Some interrelated aspects of the study of texts seem particularly relevant to exemplify their complexity and openness with respect to modelling in Digital Humanities (Ciula and Marras 2016): dynamicity, emerging particularly in the relationship between models and objects as well models and interpretations; multidimensionality, emerging from the relation between objects and interpretations; historicity, putting models in relation to their historical interpretations; processuality, playing at the interface between objects and subject's interpretations. The term dynamicity relates to the sociology of texts theory as well as the overarching principle of fluid textuality (for instance, with respect to the modelling of many variants of material texts) and engagement with hybrid modalities (for example, encompassing maps and narrative drawings). 'Multidimensionality' stands for a pluralistic model of text (Sahle 2006 and 2012) whereby the definition of text depends on how modellers look at it, on the aspects we are most interested in making explicit in our modelling efforts and the tacit knowledge invested in those efforts. The concept of historicity is useful to argue that, generally, in Digital Humanities, modelling acts are also and necessarily meta-modelling activities, because they explicitly engage with objects mediated by prior interpretative activities even if not always passing via computational formalisations. ${ }^{16}$ Therefore, our models of texts also embed historical understandings, categorisations and definitions of those texts. Last but not least, the concept of processuality aims at highlighting a further connection between the reading (and hence also modelling) of texts and our making sense of ourselves. How we read, interpret, engage with texts is intimately situated in the performative and eventful process of creating and confirming our own identity of human beings (Meister 2007).

This brief encounter with texts aimed to reiterate how entangled any Digital Humanities modelling process is with the cultural, historical and personal engagement we have and had with these complex and open objects.

In Ciula and Marras (2016), we reflected further on the importance of an imaginative use of language in Digital Humanities (modelling language, language of representation or language as vehicle of theories and methods) - for instance via the coinage of neologisms as well as the adoption of metaphors to structure knowledge - to exemplify how the terminology being chosen and the relevant metaphoric models being applied organize the modelling processes themselves.

Now I ask, if modelling is in principle an open practice to manipulate cultural (textual) objects, to read and re-read them by creating new or reinstating old interpretations, and to repurpose the work of the past, in what ways are our digital models creating memory and knowledge? Will they be legible as data as well as knowledge in the future? How is the different materiality between objects and models reconciled? Nowviskie raises an important issue of 'illegibility' of works produced as part of research projects in Digital Humanities:

16. Interestingly, Knox (2012) states that "historical modeling is necessarily metamodeling. If the passage of time has done anything interesting with the original model, we can neither simply reproduce it nor ignore it. If that past model has changed, or is inconsistent, or incoherent, or unintelligible, we may be able to correct it, or we may need to incorporate it and model its difficulties and historicity as well, depending on what we are trying to accomplish." 
Products of digital work in the humanities are evident all around us, but the arguments that they instantiate remain deceptively tacit to those who have not learned to appreciate their sites of discourse, their languages and protocols. Humanities-computing arguments are made collectively and tested iteratively. The field advances through craft and construction: the fashioning and refashioning of digital architectures and artifacts. It is little wonder that bibliographers, archivists and textual critics, and archaeologists and other specialists in material culture were the first to grasp the implications of digital technology for humanities scholarship. Methodological, embodied, and quiet knowledge transfer lies at the heart of our work, which can remain frustratingly illegible to scholars whose experience rests more in verbal exchange. (Nowviskie 2012)

However, is this illegibility a problem confined to those that are not familiar with Digital Humanities way of arguing via digital products or is a more fundamental issue? McGann used the critical edition as a focus of his reflections on the primacy of philology in the digital age. By philology he means explicitly "a science of archival memory" (McGann 2014, 41), "a research method, a science, for preserving a practical memory of the importance of memory" (McGann 2014,47 ) and he adds that the main problem is not to save our paper-based documentary record destined mostly to be lost, but rather "what we will choose to save, and why, and how will we do it" (McGann 2014, 30) and hence how we will transform "storage into memory, and data into knowledge" (McGann 2014, 96).

I conclude by stating that my take on how best to make "machines of information" which are also "machines of reflection" (McGann 2014, 95) is to study and study again the dynamic condition of texts as open objects (with respect to modelling), but also to embrace a semiotic model of modelling able to grasp these dynamic aspects. This means to me to create digital models that enact practical memory and envision a Digital Humanities which is of the Humanities, hence inherently and consciously subjective.

\section{References}

4Humanities. 4Humanities. Accessed July 21, 2016. http://4humanities.org/.

Bradley, J. (2015). How about Tools for the whole range of scholarly activities? Paper presented at Digital Humanities 2015, University of Western Sydney, Australia, June 29-July 3, 2015. http://oapen.org/download?type=document\&docid $=429194$.

Bod, R. (2014). How the humanities changed the world. OUPblog, February 17. Accessed July 21, 2016. http://blog.oup.com/2014/02/how-the-humanities-changed-the-world/.

Bod, R.; Maat, J. \& Weststeijn, T. (eds.) (2012). The making of the humanities. vol. II: from early modern to modern disciplines. Amsterdam: Amsterdam University Press. Print and Web. Accessed July 21, 2016. http://oapen.org/download?type=document\&docid=429447.

Bod, R.; Maat, J. \& Weststeijn, T. (eds.) (2010). The making of the humanities. vol. I: the humanities in early modern europe. Amsterdam: Amsterdam University Press. Print and Web. Accessed July 21, 2016. 
Ciula, A. \& Eide, Ø. (2017). Modelling in digital humanities: signs in context. Digital Scholarship in the Humanities, 32 (1): 133-146. Accessed September 7, 2017. https://doi.org/10.1093/llc /fqw045

Ciula, A. \& Eide, Ø. (2014). Reflections on cultural heritage and digital humanities: modelling in practice andt. Proceedings of the First International Conference on Digital Access to Textual Cultural Heritage - DATeCH '14: 35-41. New York, NY, USA: ACM.

Ciula, A. \& Lopez, T. (2009). Reflecting on a dual publication: Henry III Fine Rolls print and web. Literary and Linguistic Computing, 24 (2): 129-141.

Ciula, A. \& Marras, C. (2016). Circling around texts and language: towards "pragmatic modelling" in digital humanities. Digital Humanities Quarterly, 10 (3). Accessed July 21, 2016. www.digitalhumanities.org/dhq/vol/10/3/000258/000258.html

Dourish, P. (2014). NoSQL: The Shifting Materialities of Database Technology. Computational Culture, 4. Accessed September 7, 2017. http://computationalculture.net/article/no-sql-theshifting-materialities-of-database-technology

Drucker, J. (2012). Humanistic theory and digital scholarship. In Matthew K. Gold (ed.), Debates in Digital Humanities. University of Minnesota Press. Accessed July 21, 2016. http://dhdebat es.gc.cuny.edu/debates/text/34.

ESF-COST. (2013). Cultural literacy in Europe today. Science Policy Briefing, 48. Accessed July 21, 2016. www.esf.org/fileadmin/Public_documents/Publications/spb48_Cultural_Literacy.p df.

Gold, M. K. (2012). The digital humanities moment. In M. K. Gold (ed.), Debates in Digital Humanities. University of Minnesota Press. Accessed July 21, 2016. http://dhdebates.gc.cuny.e $\mathrm{du} / \mathrm{deb}$ ates/text/2.

International Conference Rethinking Humanities. December 10-11, 2015. Accessed July 21, 2016. http://labcom-ifp.ubi.pt/event/2965

Karstens, B. (2012). Bopp the builder. Discipline formation as hybridization: the case of comparative linguistics. In R. Bod, J. Maat \& T. Weststeijn (eds.), The making of the humanities. vol. II: from early modern to modern disciplines (pp. 103-128). Amsterdam: Amsterdam University Press. Print and Web. Accessed July 21, 2016. http://oapen.org/download?type=docume nt\&docid $=429447$.

Kralemann, B. \& Lattmann, C. (2013). Models as icons: modeling models in the semiotic framework of Peirce's theory of signs. Synthese, 190 (16): 3397-3420.

Knox, D. (2012). What is the thing that changes?: Space and time through the atlas of historical county boundaries. Paper presented at the workshop Knowledge Organization and Data Modeling in the Humanities: An ongoing conversation, Brown University, RI, March 16, 2012. Accessed July 21, 2016. www.wwp.northeastern.edu/outreach/conference/kodm2012/knox/ knox_paper.pdf

Meister, J. C. (2007). Events are us. Amsterdam International Electronic Journal for Cultural Narratology, Autumn, 4. Accessed July 21, 2016. http://cf.hum.uva.nl/narratology/a07_meister. htm 
McCarty, W. (2012). The residue of uniqueness. In M. Thaller (ed.), Controversies around the digital humanities (pp. 24-45). Historical Social Research, 37(3).

McGann, J. (2014). A new republic of letters: memory and scholarship in the age of digital reproduction. Harvard University Press.

Nowviskie, B. (2012). Praxis, through prisms: a digital boot camp for grad students in the humanities. The Chronicle of Higher Education, 58 (35). Accessed July 21, 2016. http://chronicle.co m/article/A-Digital-Boot-Camp-for-Grad/131665/

Olteanu, A. (2015). Philosophy of education in the semiotics of Charles Peirce. A cosmology of learning and loving. Oxford, Bern, Berlin, Bruxelles, Frankfurt am Main, New York, Wien: Peter Lang.

Palmer, C. L. (2005). Scholarly work and the shaping of digital access. Journal of the American Society for Information Science and Technology, 56 (11): 1140-1153.

Sahle, P. (2006). What is text? A pluralistic approach. Digital Humanities 2006 Conference Abstracts, 188-190. Université Paris-Sorbonne, CATI, July 5-9.

Sahle, P. (2012). Modeling transcription. Paper presented at the workshop Knowledge Organization and Data Modeling in the Humanities: An ongoing conversation, Brown University, RI, March 15. Accessed July 21, 2016. https://datasymposium.wordpress.com/sahle/

Small, H. (2013). The value of the humanities. Oxford: Oxford University Press.

Smithies, J. (2017). The digital humanities and the digital modern. Palgrave Macmillan UK.

Svensson, P. (2012). Envisioning the digital humanities. Digital Humanities Quarterly 6 (1). 